\title{
Zur Frage der Wirkung einer Anaesthesie auf den Steroidstoffwechsel in der Leber
}

\author{
Von J. Breuer, E. Paulus und H. Breuger \\ Institut für Klinische Biochemie und Klinische Chemie der Universität Bonn
}

(Eingegangen am 27. Juli/14. September 1973)

Der in-vitro-Stoff wechsel von $\left[4-{ }^{14} \mathrm{C}\right] \mathrm{O}$ stron wurde in Leberschnitten unbehandelter und anacsthesierter Ratten untersucht. Weder eine zweistündige Neuroleptanalgesie alleine noch die zusätzliche Gabe eines Muskelrelaxans und Beatmung mit $\mathrm{N}_{2} \mathrm{O} / \mathrm{O}_{2}$ beeinflußten den Umsatz von $\left[4-{ }^{14} \mathrm{C}\right]$ Ostron sowie die Bildung von $\left[4-{ }^{14} \mathrm{C}\right]$ Ostradiol-17 $\beta$ und von hydroxylierten Metaboliten. Daraus wird der Schluß gezogen, daß die Aussagekraft von in-vitro-Versuchen mit Gewebe, das intra operationem gewonnen wurdc, nicht eingeschränkt ist.

\section{On the effect of anaestbesia on the metabolism of steroids in the liver}

The metabolism in vitro of $\left[4{ }^{14} \mathrm{C}\right]$ oestrone was studied in liver slices of untreated and of anaesthesised male Wistar rats. Neither neuroleptanalgesia alone nor the additional administration of a muscular relaxant and artificial respiration with $\mathrm{N}_{2} \mathrm{O} / \mathrm{O}_{2}$ during two hours had any influence on the rate of the metabolism of $\left[4-{ }^{14} \mathrm{C}\right]$ oestrone, the formation of $\left[4-{ }^{14} \mathrm{C}\right]$ oestradiol- $17 \beta$, or the formation of hydroxylated metabolites. It is concluded that the results of in vitro experiments performed with tissue, obtained during operation, are not affected by anaesthesia.

Die Untersuchung des in-vitro-Stoffwechsels von Steroidhormonen beim Menschen erfolgt fast ausschließlich an Gewebeproben, die intra operationem gewonnen werden. In diesem Zusammenhang erhebt sich die Frage, ob eine Anaesthesie die Aktivität von steroidmetabolisierenden Enzymen beeinflußt. Eine solche Wirkung könnte durch Aktivierung, durch Hemmung und/oder durch Induktion von Enzymen stattfinden. Im Hinblick auf die zum Teil weitreichenden Interpretationen der qualitativen und quantitativen Ergebnisse von in-vitro-Studien wurde in der vorliegenden Arbeit versucht, die oben gestellte Frage zu beantworten. Die Untersuchungen konnten aus naheliegenden Gründen nicht am Menschen durchgeführt werden; deshalb wurden Ratten verwendet, deren Steroidstoffwechsel demjenigen des Menschen ähnlich ist (vgl. (1)). Außerdem wurde eine Narkoseform verwendet, wie sie in der Humanmedizin häufig gebraucht wird.

\section{Methodik}

\section{Steroide}

[4-14 C]Östron (3-Hydroxy-1,3,5(10)-östratrien-17-on; spez. Aktivität $50,0 \mathrm{mCi} / \mathrm{mmol}$ ) wurde vom Radiochemical Centre, Amersham, England, bezogen; vor dem Gebrauch wurde [4-14 $\mathrm{C}]$ Östron durch Chromatographie auf formamidimprägniertem Papiet mit Monochlorbenzol als Laufmittel gereinigt. Ostron, Ostradiol$17 \beta(1,3,5(10)$-Östratrien-3,17 $\beta$-diol) und Óstriol (1,3,5(10)-Östra-

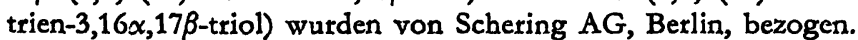

\section{Reagenzien und Lösungsmittel}

Alle organischen Lösungsmittel wurden vor Gebrauch destilliert; die verwendeten Reagenzien waten von p. a. Reinheitsgrad (Merck, Darmstadt).
Tiere und Gcwebe

Als Versuchstiere dienten männliche Wistar-Ratten im Alter von 8-10 Wochen (Gewicht 300-350 g). Die Tiete wurden durch Dekapitation getötet und die Lebern nach dem Ausbluten entnommen. Die Gewebsschnitte wurden nach Deutsch (2) hergestellt.

\section{Inkubationsbedingungen}

Die Inkubationsversuche wurden unter optimalen Reaktionsbedingungen durchgeführt. Diese Reaktionsbedingungen waren in kinetischen Untersuchungen unter Berücksichtigung des $\mathrm{pH}$ Wertes, der Inkubationsdauer, der Gewebemenge und der Substratmenge vorher ermittelt worden. Jeweils $100 \mu \mathrm{g}\left[4-{ }^{14} \mathrm{C}\right]$ Östron $(0,2 \mu \mathrm{Ci})$ wurden mit $100 \mathrm{mg}$ Leberschnitten in $5,0 \mathrm{ml}$ glucosehaltigem (20 mmol/l) KREBS-RINGER-Phosphatpuffer, $\mathrm{pH}$ 7,4 , bei $37^{\circ} \mathrm{C} 120 \mathrm{~min}$ in einem Schüttelthermostaten unter Luft inkubiert. Das Steroid wurde in Propylenglycol $(4 \mathrm{mg} / \mathrm{ml})$ den Inkubationsansätzen zugesetzt; die Endkonzentration von Propylenglycol betrug $0,5 \%$.

\section{Aufarbeitung der Versuchsansätze}

Nach Beendigung der Versuche wurden die Inkubationsansätze dreimal mit je $10 \mathrm{ml}$ wassergesättigtem Essigsäureäthylester extrahiert und die vereinigten Extrakte unter Stickstoff im Rotationsverdampfer eingedampft. Die Extraktrückstände wurden in $0,3 \mathrm{ml}$ Methanol aufgenommen.

\section{Papierchromatographie}

Es wurde auf Schleicher-\&-Schüll-Papicr 2043b $\mathrm{Mgl}$ bei $25^{\circ} \mathrm{C}$ absteigend chromatographiert. Alle Trockenrückstände wurden zunächst der Chromatographie auf formamidimprägniertem Papier mit Monochlorbenzol unterworfen; die „Ostriol“-Fraktion wurde auf formamidimprägniertem Papier mit Chloroform rechromatographiert. Die phenolischen Steroide, die als Testverbindungen dienten, wurden mit Folin-Crocalteus-Reagenz (3) sichtbar gemacht.

\section{Quantitative Bestimmung}

Die quantitative Bestimmung der phenolischen Steroide erfolgte auf den Papierchromatogrammen durch Messung der Radio- 
aktivität mit einem Radiopapierchromatographen (Friesecke \& Höpfner, Erlangen) unter Verwendung eines fensterlosen MethanDurchflußzählers mit 4- $\pi$-Zählgeometrie $(14 \%$ Zählausbeute für $\left.{ }^{14} \mathrm{C}\right)$. In einigen Vergleichsuntersuchungen wurde $\left[4-{ }^{14} \mathrm{C}\right] \mathrm{O}$ stron von den Papierchromatogrammen eluiert und die methanolische Lösung zur Trockene eingedampft. Der Rückstand wurde in $12 \mathrm{ml}$ einer Szintillations-Lösung, enthaltend $5 \mathrm{~g}$ 2,5-Diphenyloxazol (PPO) und 0,3 g 1,4-Bis(4-methyl-5-phenyl-2-oxazolyl)benzol (Dimethyl-POPOP)/1 Toluol, gelöst und die Radioaktivität im Packard-Tri-Carb-Szintillations-Spektrometer (Modell 3003) unter Verwendung eines externen Standards gemessen. Die Zählausbeute für ${ }^{14} \mathrm{C}$ betrug $80 \%$.

\section{Durchführung der Anaesthesie}

Neuroleptanalgesie: Die Tiere erhielten zu Beginn der Narkose und nach $45 \mathrm{~min}$ intraperitoneal jeweils $0,02-0,03 \mathrm{mg} / \mathrm{kg}$ Körpergewicht Fentanyl (1-N-2-Phenaethyl-4-N-propionyl-anilinopiperidin-dihydrogencitrat) und $1,25-1,50 \mathrm{mg} / \mathrm{kg}$ Körpergewicht Dehydrobenzperidol (1-[3-(4-Fluot-benzoy1)-propyl]-4-(2-oxo-1benzimidazolinyl)-1,2,3,6-tetrahydropyridin). Neuroleptanalgesie bei gleichzeitiger Muskelrelaxation und Intubation eines $\mathrm{N}_{2} \mathrm{O} / \mathrm{O}_{2}-$ Gemisches: Neben den oben angegebenen Neuroleptanalgetica wurde den Tieren alle $15 \mathrm{~min}$ jeweils $1 \mathrm{mg} / \mathrm{kg}$ Körpergewicht Suxamethonium (Bernsteinsäure-bis-cholinester) intraperitoneal injiziert. Die vollständig relaxierten Tiere wurden mit einem Gemisch von $\mathrm{N}_{2} \mathrm{O} / \mathrm{O}_{2}(2,5: 1)$ beatmet. Die Beatmung erfolgte nach Tracheotomie und Einsetzen eines kleinen Tubus mit Hilfe eines Narkoseapparates.

Alle Tiere wurden $120 \mathrm{~min}$ anaesthesiert und anschließend sofort getötet.

\section{Ergebnisse und Diskussion}

Unter den hier gewählten Inkubationsbedingungen wurden im Mittel $60 \%$ der eingesetzten Menge von [4-14 C]Östron durch Leberschnitte männlicher WistarRatten metabolisiert (vgl. Tab. 1). Unter den Metaboliten wurden zwei Fraktionen papierchromatographisch nachgewiesen: $\left[4-{ }^{14} \mathrm{C}\right]$ Östradiol-17 $\beta$ entstand zu etwa 3\%, während die „Östriol"-Fraktion etwa $23 \%$ ausmachte. $\left[4-{ }^{14} \mathrm{C}\right]$ Östradiol-17 $\beta$ war papierchromatographisch einheitlich und wurde nicht weiter identifiziert, zumal es sich bei diesem Reduktionsprodukt um einen bekannten Metaboliten von Östron handelt. Die „Östriol"-Fraktion enthält nach 1. c. (1) $6 \alpha, 6 \beta-, 7 \alpha-, 16 \alpha-$ und 16ק-Hydroxyöstron, $6 \alpha-, 6 \beta-$ und $7 \alpha$-Hydroxyöstradiol-17 $\beta$ sowie 16 -Epiöstriol. Auf eine vollständige Auftrennung der „Östriol“-Fraktion sowie die quantitative Bestimmung der einzelnen Metaboliten wurde verzichtet, da im vorliegenden
Zusammenhang die Gesamthydroxylierungsrate von Wichtigkeit war. Die an den zehn Kontrolltieren ermittelten quantitativen Ergebnisse für $\left[4{ }^{14} \mathrm{C}\right]$ Östron, $\left[4-{ }^{-14} \mathrm{C}\right]$ Östradiol-17 $\beta$ und die „Östriol ${ }^{\varsigma 6}-$ Fraktion stimmen im wesentlichen mit den Befunden früherer Arbeiten überein $(4,5)$.

Wie aus der Tabelle weiter hervorgeht, hatte eine Neuroleptanalgesie mit Thalamonal (Fentanyl und Dehydrobenzperidol) während zwei Stunden keinen erkennbaren Effekt auf den Umsatz von $\left[{ }^{14} \mathrm{C}\right]$ Östron sowịe die Bildung von $\left[{ }^{14} \mathrm{C}\right]$ Östradiol-17 $\beta$ und die Hydroxylierung der beiden Östrogene zu polaren Metaboliten. Auch unter den Bedingungen einer Neuroleptanalgesie in Kombination mit einem Muskelrelaxans und Beatmung mit $\mathrm{N}_{2} \mathrm{O} / \mathrm{O}_{2}$ (Dauer ebenfalls zwei Stunden) konnte keine Wirkung auf den Umsatz, die Reduktion und die Hydroxylierung von $\left[4^{-14} \mathrm{C}\right]$ Östron nachgewiesen werden.

Die in der vorgelegten Arbeit angewandte Neuroleptanalgesie entspricht - auch in der Dosierung der in der Humanmedizin üblichen Narkoseform (vgl. $6,7)$. Wie bereits eingangs erwähnt, bestehen hinsichtlich des Intermediärstoffwechsels von Östrogenen zwischen der Rattenleber und der Leber des Menschen keine wesentlichen Unterschiede; die enzymatischen Reaktionen sind weitgehend identisch (vgl. 1). Diese Feststellung sowie die hier erhobenen Befunde lassen - bei aller gebotenen Vorsicht - den Schluß zu, daß der in-vitro-Stoffwechsel von Steroidhormonen in menschlichem Gewebe durch eine Anaesthesie nicht beeinflußt wird; infolgedessen dürfte die Aussagekraft von in-vitro-Versuchen durch die Tatsache, daß das Gewebe intra operationem entnommen wurde, nicht eingeschränkt sein. Abschließend sei erwähnt, daß TornetTA und Boger (8) beim Menschen 5 Tage nach einer Anaesthesie mit Fentanyl und Dehydrobenzperidol (INN: Droperidol) ebenfalls keine Veränderung verschiedener Leberfunktionsproben (z. B. alkalische Phosphatase, Lactatdehydrogenase, Aspartat- und Alanintransaminase sowie Bromsulfphthaleintest) gefunden haben. Die häufig beobachtete Induktion von 'Lebetenzymen durch Pharmaka (vg1. 9) scheint bei einer zeitlich begrenzten Narkose keine wesentliche Rolle zu spielen.

Tab. 1

Stoffwechsel von [4-1"C]Östron in Leberschnitten normaler und unter verschiedenen Bedingungen anaesthesierter männlicher Wistar-Ratten. Jeweils $100 \mu \mathrm{g}$ [4-1' $\mathrm{C}$ ] Ostron wurden mit $100 \mathrm{mg}$ Leberschnitten in $5,0 \mathrm{ml}$ glucosehaltigem KREBS-RINGER-Phosphatpuffer, $\mathrm{pH} \mathrm{7,4,}$, bei $37^{\circ} \mathrm{C}$ unter Luft $120 \mathrm{~min}$ inkubiert. Weitere Einzelheiten über die Anaesthesie und die Inkubationsversuche vgl. Methodik

Die Werte in der Tabelle sind Mittelwerte \pm Standardabweichung von jeweils 10 Tieren; alle Bestimmungen wurden als Doppelbestimmungen durchgeführt. In Klammern sind die höchsten und niedrigsten Werte für die einzelnen Gruppen angegeben

$E_{1}=\left[4-{ }^{14} \mathrm{C}\right]$ Ostron, $\mathrm{E}_{2}=\left[{ }^{4-14} \mathrm{C}\right]$ Ostradiol-17 $\beta, \mathrm{E}_{3}=$ „Ostriol"-Fraktion (polare Metaboliten)

\begin{tabular}{|c|c|c|c|c|}
\hline $\begin{array}{l}\text { Experimentelle } \\
\text { Bedingungen }\end{array}$ & Zahl der Tiere & $\underset{\mu \mathrm{g}}{\mathrm{E}_{1} \text { umgesetzt }}$ & $\begin{array}{c}\mathrm{E}_{2} \text { gebildẹt } \\
\mu \mathrm{g}\end{array}$ & $\begin{array}{c}E_{3} \text { gebildet } \\
\mu \mathrm{g}\end{array}$ \\
\hline Kontrollen & 10 & $\begin{array}{r}60 \pm 9,8 \\
(43-75)\end{array}$ & $\begin{array}{c}3,0 \pm 1,1 \\
(1,6-5,0)\end{array}$ & $\begin{array}{r}23 \pm 4,2 \\
(17-33)\end{array}$ \\
\hline Neuroleptanalgesie & 10 & $\begin{array}{r}56 \pm 8,1 \\
(36-74)\end{array}$ & $\begin{array}{c}3,4 \pm 1,2 \\
(2,5-4,2)\end{array}$ & $\begin{array}{r}19 \pm 3,5 \\
(15-30)\end{array}$ \\
\hline $\begin{array}{l}\text { Neuroleptanalgesie } \\
+\mathrm{Muskelrelaxans} \\
+\mathrm{N}_{2} \mathrm{O} / \mathrm{O}_{\mathrm{a}}\end{array}$ & 10 & $\begin{array}{r}64 \pm 7,0 \\
(47-73)\end{array}$ & $\begin{array}{c}3,1 \pm 1,1 \\
(1,4-4,2)\end{array}$ & $\begin{array}{r}18 \pm 5,7 \\
(11-31) \\
:\end{array}$ \\
\hline
\end{tabular}




\section{Literatur}

1. BREUER, H. \& KNUPPEN, R. (1969), in: Methods in Enzymology, Vol. XV, ed. Clayroon, R. B., Academic Press, New York, 691-735. - 2. Deutsch, W. (1936), J. Physiol. 87, 56 P. 3. Folin, O. \& Crocaltev, V. (1927), J. Biol. Chem. 73, 627-635. - 4. BreUER. H., NoCkE, I. \& KNUPPEN, R. (1959), Hoppe-Seylers Z. Physiol. Chem. 315, 72-79. - 5. BreUER, H., Nocke, L. \& Pangels, G. (1960), Acta Endocrinol. (Copen- hagen) 34, 359-365. - 6. STöcKER, L. (1967), Narkose, Thieme, Stuttgart. - 7. AllGÖWER, M. (1971), Allgemeine und spezielle Chirurgie, Springer, Berlin-Heidelberg-New York. - 8. TorNETTA, F. J. \& BOGER, W. P. (1964), Anaesthesia and Analgesia Current Researches 43, 544-559. - 9. Remmer, H. (1972), Eur. J. Clin. Pharmacol. 5, 116-136.
Prof. Dr. H. Breuer Inst. für Klin. Biochemie 53 Bonn-Venusberg 